\title{
Extensive Fetal Congenital Subcutaneous Mixed Venous Lymphatic Lesion: Prenatal Diagnosis and Postnatal Management
}

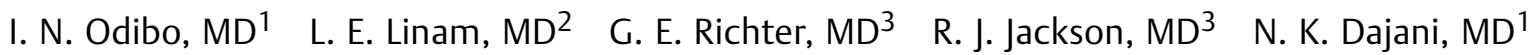 \\ ${ }^{1}$ Department of Obstetrics and Gynecology, University of Arkansas for \\ Address for correspondence Imelda N. Odibo, MD, 4301 W. Markham \\ Medical Sciences, Arkansas \\ 2 Department of Radiology, Arkansas Children's Hospital, Arkansas \\ St., \#518, Little Rock, AR 72205 (e-mail: inodibo@uams.edu). \\ 3 Department of Otolaryngology, Arkansas Children's \\ Hospital, Arkansas \\ Am J Perinatol Rep 2015;5:e37-e42.
}

\begin{abstract}
Vascular lesions may be categorized as proliferative tumors, such as hemangiomas, or nonproliferative malformations that include capillary, lymphatic, venous, arterial, or

Keywords

- congenital disorders

- pediatrics

- pregnancy

- obstetrics and gynecology

- maternal fetal medicine

- venous lymphatic lesions mixed lesions. Lymphatic malformations are benign localized congenital malformations of the lymphatic system. They may be microcystic or macrocystic lesions or a combination of both. The lesions may also be uniseptate or multiseptate, and are more commonly located in the head and neck or axillary region. Prenatal diagnosis is based on ultrasound and magnetic resonance imaging. Postnatal management largely depends on the size and location of the lesion. This is the first case report of prenatally diagnosed extensive subcutaneous macrocystic venous lymphatic malformation involving the fetal thorax, back, pelvis, and lower extremities. Prenatal course and postnatal management are described. This report will aid other specialists in the field of prenatal diagnosis and postnatal surgery in the evaluation and management of these patients.
\end{abstract}

\section{Summary}

Vascular lesions may be categorized as proliferative tumors, such as hemangiomas, or nonproliferative malformations that include capillary, lymphatic, venous, arterial, or mixed lesions. ${ }^{1}$ Lymphatic malformations are benign localized congenital malformations of the lymphatic system. They may be microcystic or macrocystic lesions or a combination of both. The lesions may also be uniseptate or multiseptate, and are more commonly located in the head and neck or axillary region. Prenatal diagnosis is based on ultrasound (US) and magnetic resonance imaging (MRI). Postnatal management largely depends on the size and location of the lesion.

This is the first case report of prenatally diagnosed extensive subcutaneous macrocystic venous lymphatic malformation involving the fetal thorax, back, pelvis, and lower extremities. Prenatal course and postnatal management are described. This report will aid other specialists in the field of prenatal diagnosis and postnatal surgery in the evaluation and management of these patients.

\section{Case Presentation}

A 31-year-old female, gravida 1, para 0, was referred to the Arkansas Reproductive Genetics Program (ARGP) at 19 weeks' gestation for possible gastroschisis. Maternal history was significant for tobacco use and heavy alcohol consumption in the first trimester. Ultrasonography revealed no evidence of gastroschisis. There were, however, multiple subcutaneous fluid-filled cysts along the fetal left axilla, left lateral thorax, and anterior abdominal wall (-Fig. 1). These subcutaneous cysts may have been mistaken for herniated fetal bowel of a gastroschisis malformation. There were no vascular or solid components within the cysts. The left lower extremity appeared larger than the right lower extremity and the toes appeared widespread. received

July 24, 2014

accepted after revision

December 4, 2014

published online

February 25, 2015
DOI http://dx.doi.org/

10.1055/s-0034-1544107. ISSN 2157-6998.
Copyright $\odot 2015$ by Thieme Medical Publishers, Inc., 333 Seventh Avenue, New York, NY 10001, USA. Tel: +1(212) 584-4662.
License terms

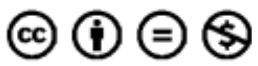


The patient was offered but declined both amniocentesis and cell-free fetal DNA testing. She was counseled about the poor prognosis given the extensive nature of the lesions and potential for development of nonimmune hydrops. The patient was followed with serial US imaging throughout the pregnancy. The cystic lesions, which were initially noted on the left lateral axilla and thorax, extended to the pelvis and progressed through the back to the right aspect of the thorax by 31 weeks. Measurements of the mass were $9.6 \times 7.7 \times 7.8 \mathrm{~cm}$ at 26 weeks, $10.1 \times 10.6 \times 9.1 \mathrm{~cm}$ at 29 weeks, and peaked at $14.0 \times 11.6 \times 9.5 \mathrm{~cm}$ at 31 weeks. Intra-abdominal development of lymphatic cysts was suspected with the appearance of a small cystic lesion adjacent to the right kidney. A fetal MRI at 29 weeks' gestation confirmed an extensive cystic mass extending from the fetal ear to the pelvis, this mass measured $16.5 \mathrm{~cm}$ craniocaudal by $10.6 \mathrm{~cm}$ anterior posterior by $7.5 \mathrm{~cm}$ transverse ( - Fig. 2). In addition to the subcutaneous portion, cysts were seen in the retroperitoneum and in the chest, anterior to the heart (although the intrathoracic portion was not appreciated until postnatally). The airway was not involved by the lesion, and remained patent throughout its course (-Fig. 3). The cysts remained relatively stable for the rest of the pregnancy with some resolution of the cysts in the pelvic area.

Antepartum surveillance (weekly nonstress test, Doppler, and fluid assessment) was initiated at 32 weeks and remained normal. Polyhydramnios was noted at 34 weeks but was resolved by 36 weeks. The fetus had normal growth throughout gestation without evidence of hydrops, ascites or pleural effusion. Due to the size of the lesion and anticipated problems related to dystocia, a planned cesarean delivery was performed at 39 weeks. The procedure was uncomplicated and a male infant weighing 4,120 g was delivered with Apgar scores of nine and nine at 1 and 5 minutes, respectively. Umbilical artery cord gas values were normal.

Postnatal physical examination showed large skin colored soft cystic masses extending from the left axilla to the pelvis and extending through the back to the right trunk. The mass on the left axilla and thorax displaced the left arm upwards.

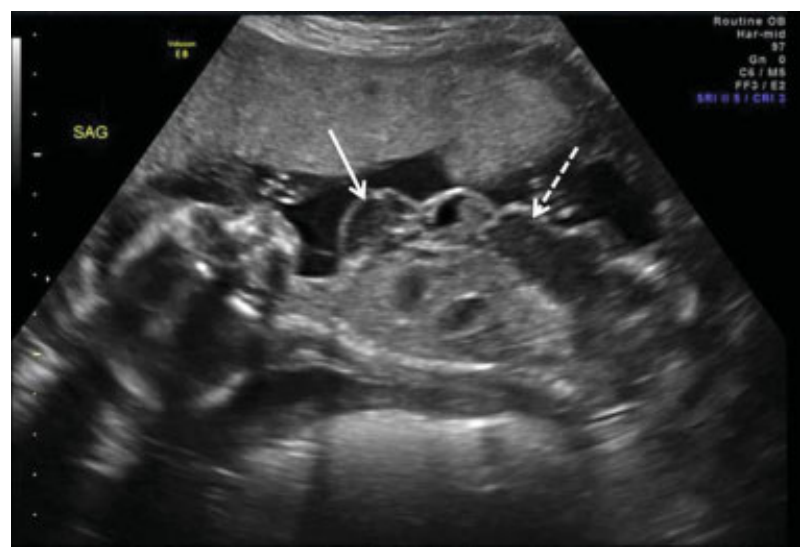

Fig. 1 Prenatal ultrasound images showing the sagittal view demonstrating anterior subcutaneous lesions. Cyst over chest (solid arrow). Cyst over abdominal wall (dashed arrow).

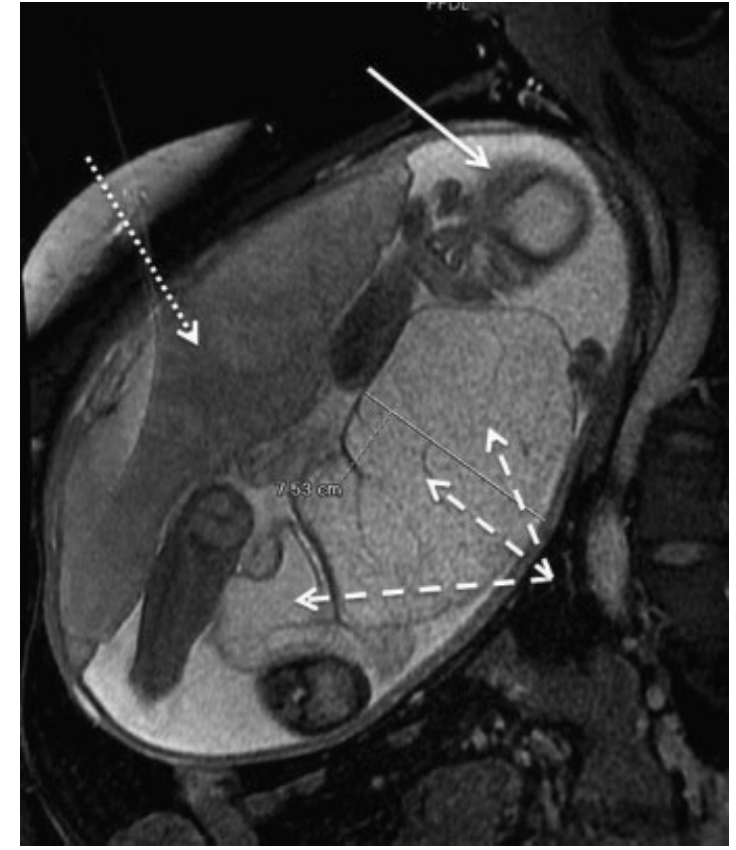

Fig. 2 Coronal and axial fast imaging employing steady-state acquisition and coronal fetography imaging show a multiloculated, high $\mathrm{T} 2$ signal mass involving the subcutaneous tissues of the trunk. This mass measures $7.53 \mathrm{~cm}$ transverse by $10.4 \mathrm{~cm}$ anterior posterior by $18.48 \mathrm{~cm}$ craniocaudal.

Multiple irregular areas of skin discoloration were noted on the mass at different locations with the largest lesion on the left. There were areas of redundant skin folds in the pelvic area, which may represent the partial resolution of the cystic

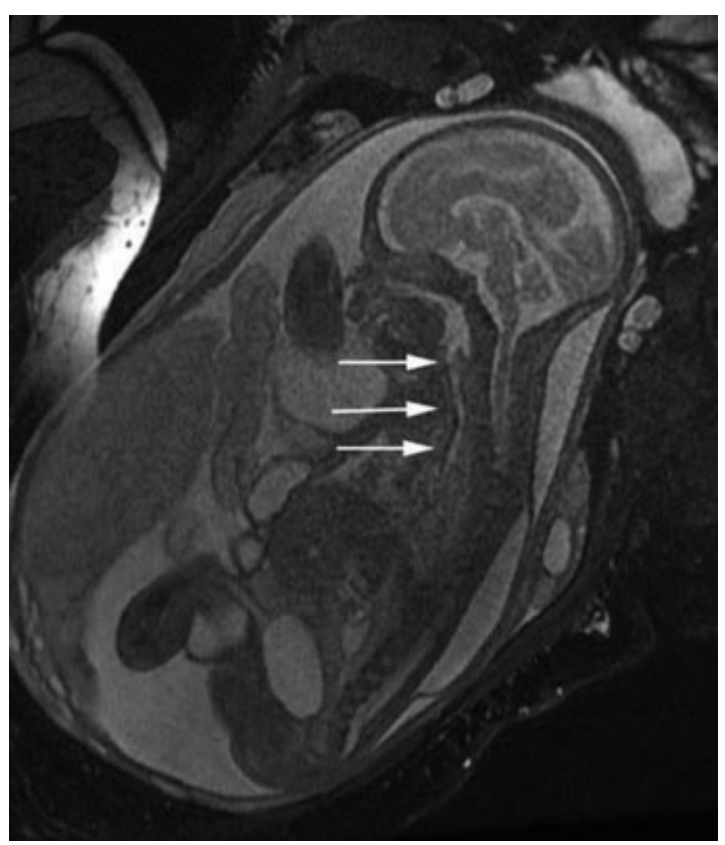

Fig. 3 Sagittal fast imaging employing steady-state acquisition image at the level of the airway. When evaluating a lymphatic malformation involving the trunk or neck, it is important to demonstrate airway patency. This airway is normal (arrows). 
masses as noted in prenatal US in late gestation. The left lower extremity was significantly larger than the right with toes appearing widespread bilaterally. There were no gross dysmorphic facial features ( - Fig. 4A, B).

Postnatal radiographs showed extensive soft tissue swelling with small punctate calcifications in the soft tissues suggesting phleboliths. An US was performed of the malformation which showed a micro- and macrocystic lesion involving the subcutaneous tissues of the torso. Fluid-fluid levels were seen in the larger cysts, suggesting hemorrhage. Postnatal MRI of the chest, abdomen, and pelvis on day 1 of life again showed the extensive subcutaneous cystic lesion involving the entire torso. Increased T1 signal in the largest cyst was new since the prenatal MRI, suggesting hemorrhage (-Fig. 5). In addition to the subcutaneous cysts, this lesion extended into the paraspinous musculature, retroperitoneum, left pleural space, and pericardial space.

An echocardiogram was unremarkable except for a vascular versus cystic area noted at the apex of the heart. This lesion appeared to be outside of the ventricular wall but it was unclear whether it involved the pericardial sac or was external to it. Ophthalmology evaluation was unremarkable. Skeletal surveys showed a large soft tissue mass involving the torso and bilateral clubfeet deformities. Genetics evaluation led to the suspicion of CLOVES syndrome (Congenital Lipomatous Overgrowth Vascular Malformations Epidermal Nevi and Spinal/Skeletal Anomalies and/or scoliosis). Neonate was also evaluated at the vascular anomalies clinic and was diagnosed with a primarily lymphatic malformation with venous and capillary components; plans for subsequent surgical resection were made.

The neonate was discharged home on the 9th day of life and readmitted at 2 months of age for cellulitis, which was treated with clindamycin. At 4 months of age, the infant underwent an extensive almost total resection of the thoracoabdominal lesion on the left axilla, chest, abdomen, and back; spot sclerotherapy with doxycycline was also used. Pathology evaluation confirmed a mixed venous and lymphatic vascular malformation with immunostain for smooth muscle actin, highlighting the thicker muscular walls of the venous channels and D 2-40 stain showing lymphatic channels.

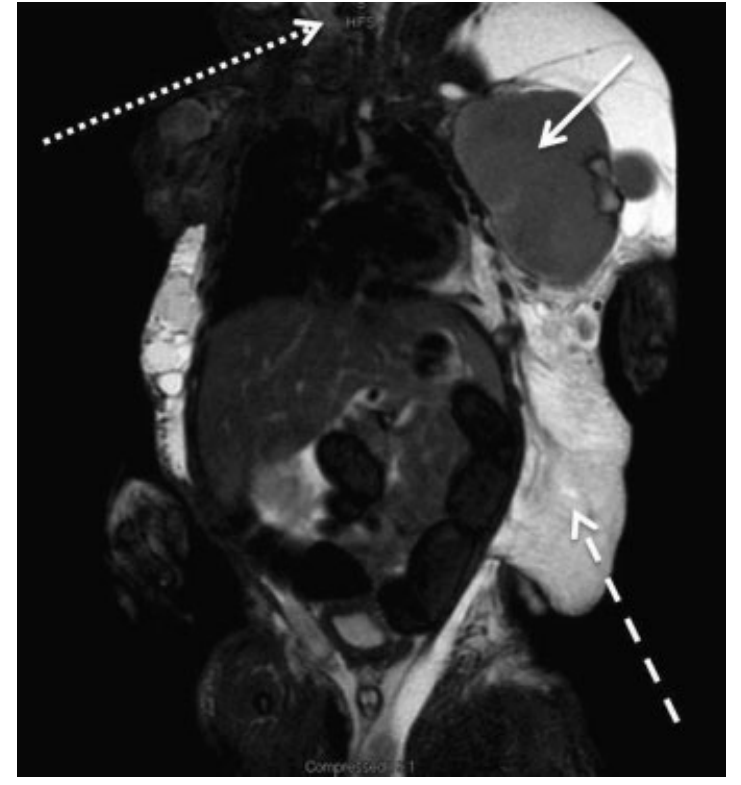

Fig. 5 Coronal T2-weighted magnetic resonance imaging 1 day of life shows the large increased T2 signal subcutaneous soft tissue mass. There is a single mass cyst with a darker signal, indicating hemorrhage (solid arrow). Torso involving subcutaneous cysts (dashed arrow). Head at the top of the image (dotted arrow).

\section{Treatment}

This patient presented with a mixed vascular malformation with a primary microcystic and macrocystic component extending along the thoracoabdominal wall bilaterally. The massive macrocystic channels were affecting normal respiration and development, and leading to failure to thrive; therefore, urgent surgical resection was chosen as the primary treatment option for this patient. In addition, it would allow for the achievement of clear planes of dissection around the malformation outside the thorax and abdominal cavities.

This patient underwent a staged resection of the external thoracoabdominal portions of the malformation. This was performed in conjunction with removal of redundant skin and involved capillary malformation of the skin ( - Fig. $\mathbf{6}$ ). The left thoracoabdominal mass was first excised at 4 months of
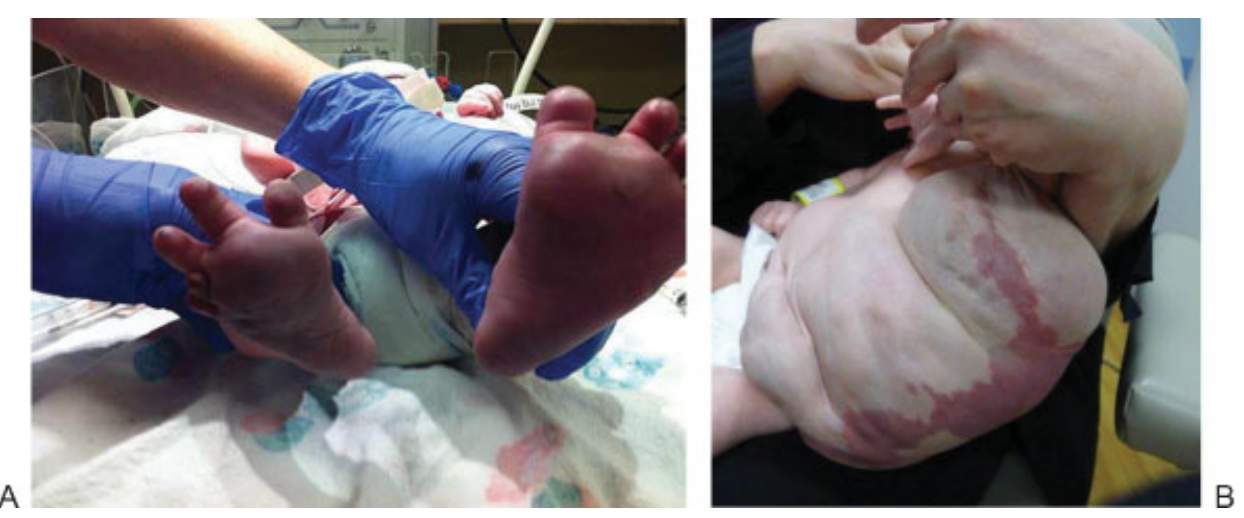

Fig. 4 (A): Postnatal view of lower extremities with left appearing larger and both with toes widespread. (B): Extensive lesions, left trunk view. 
age using a trapdoor flap extended along the pectoralis muscle, back, and inguinal canal. Preservation of normal chest and abdominal wall muscle was achieved with protection of neurovascular elements. A large macrocystic channel appeared to extend into the thorax through a small $1 \mathrm{~cm}$ defect between the 5 th and 6 th ribs. This portion of the malformation ended in a blind intrathoracic pouch. Intraoperative sclerotherapy with doxycycline was subsequently performed by infiltrating the intrathoracic malformation directly through the defect. Sclerotherapy was elected to be performed following surgical extirpation and debulking of the majority of the malformation. If performed before surgery, sclerotherapy can complicate the resection from secondary scaring and obfuscation of normal tissue planes. In the setting of extensive disease, it is best reserved for residual lesions as in our case or for recurrent lesions. The remaining malformation was dissected just below the skin to leave large subdermal flaps. Dissection proceeded from lateral to medial to the chest wall without crossing the sternum or spine to the contralateral side. Over $90 \%$ of the malformation not involving the skin was removed. Because of the risk of continued lymphatic fluid drainage, 2 penrose drains were placed and a pressure dressing was applied. The drains were removed several weeks later as the skin flaps healed.

Six months later (age 10 months), the same procedure was performed on the macrocystic malformation involving the right extrathoracic and abdominal locations. The thorax and abdomen were not entered during this procedure. Over $90 \%$ of the malformation on the right was removed from the sternal area to the spine. A curvilinear crescent-shaped excision was performed to remove some of the redundant skin and capillary malformation. A drain was also placed and removed several weeks later. Drainage of a seroma was performed 1 month following this last procedure (right resection) when the drain plugged (age 11 months). After both surgeries, the patient was maintained on oral antibiotics and steroids to reduce edema and lymphatic drainage.

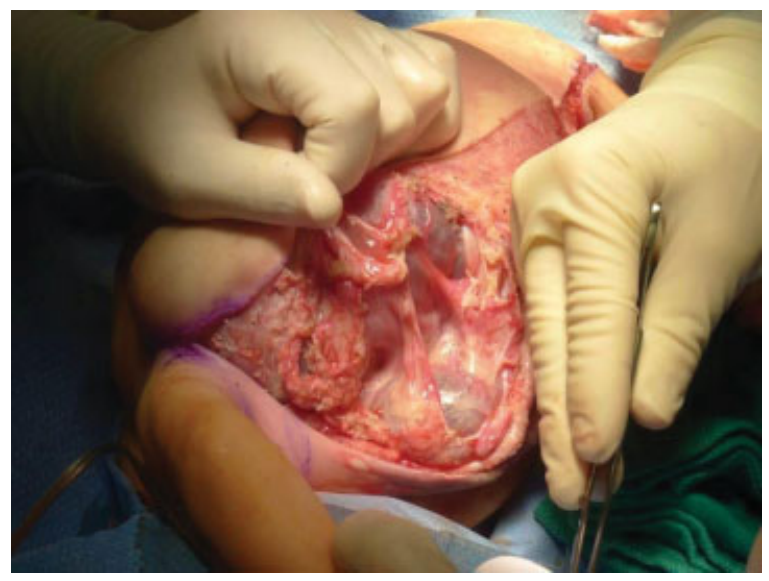

Fig. 6 Surgical procedure: Multiple cystic lesions exposed and removed from the axilla, chest, and back. Lymphatic malformation at the lower right of incision. Arm can be seen at the lower left of the image.

\section{Outcome and Follow-Up}

The child remained asymptomatic with good weight gain and activity at the age of 1 year. He has no limitation of movement, ambulates, and is achieving normal speech (- Fig. 7). No additional procedures have been required as his condition is now well controlled. He has bilateral thoracoabdominal scars that will be addressed at a later time with additional debulking of residual disease of the skin along the left pelvis and lateral chest. Laser therapy will be performed using the pulse dye laser of the capillary malformation of the skin.

\section{Discussion}

Lymphatic malformations are nonproliferative vascular malformations that occur during the embryonic stage due to obstruction of lymphatic vessels leading to the jugular venous system. The lymphatic system starts developing by the end of 5 weeks' gestation and develops as outgrowths from the venous system. Obstruction of the lymphatic vessels leading to the jugular venous system results in lymphatic malformations; this may explain the venous component in our patient. ${ }^{1,2}$ The incidence is estimated to be approximately 1.2 to $1.5 \%$ and one-fourth of these lesions is completely or partially lymphatic in origin. ${ }^{3}$ Venolymphatic malformations are more commonly located in the neck, ${ }^{4,5}$ followed by the axilla, mediastinum, and extremities. In one series, lymphatic malformations were the most commonly diagnosed vascular anomaly and occurred more commonly in the cervicofacial region, axilla, mediastinum, and extremities (in that order). ${ }^{6}$ Diagnosis has been reported as early as 16 weeks' gestation. ${ }^{6}$ Prenatal diagnosis is based mostly on two-dimensional ultrasonography. A lymphatic malformation appears as a multiloculated cystic mass, anechoic, in one or multiple locations. Hemorrhage within the cyst is usually seen as hyperechoic

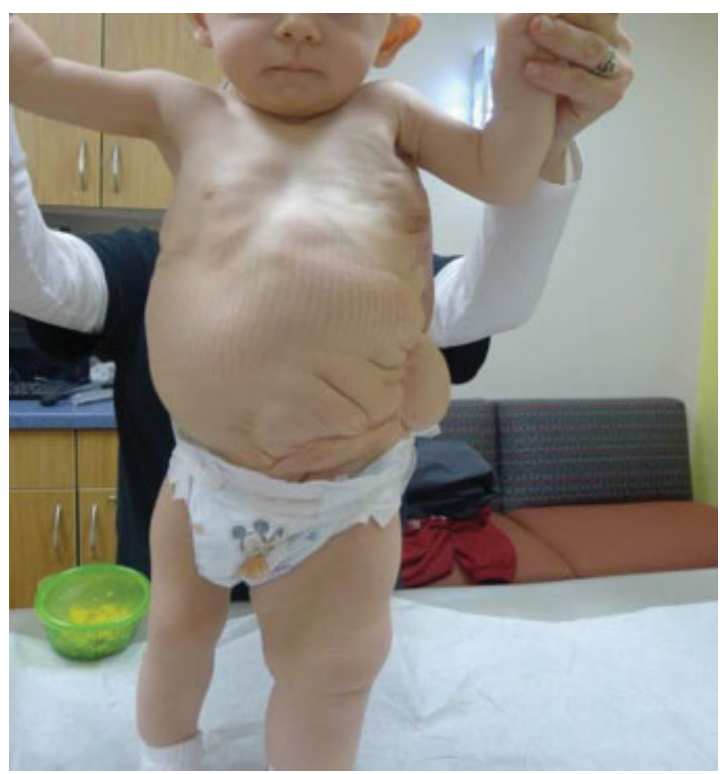

Fig. 7 Two months after the second operation, age 1 year. Front view. Left leg larger than the right. 
foci. MRI has been an important adjunct in the diagnosis of these lesions ${ }^{7,8}$ and has been used to accurately evaluate the extent and character of an extensive intra-abdominal lymphangioma and the fetal airway. ${ }^{7}$ Genetic disorders associated with the malformation include Turner syndrome, KlippelTrenaunay syndrome ${ }^{9,10}$ (capillary malformations associated with lymphatic malformations), Proteus syndrome (low-flow, complex lymphatic vascular malformations), and less commonly as part of blue rubber-bleb nevus syndrome and Maffucci syndrome, which are primary venous malformations. $^{11-13}$ CLOVES syndrome is a rare congenital disorder first described independently by Sapp et al ${ }^{14}$ and Alomari ${ }^{15}$ in 2006. Approximately 100 people have been diagnosed with it worldwide. ${ }^{16}$ For our patient, initial suspicion was high for CLOVES syndrome due to the presence of the cystic lymphatic lesions, overgrowth of the extremities, and large wide toes, but this patient did not have any ocular features or skeletal findings associated with this disorder.

Prenatal lymphatic vascular malformations may expand or contract depending on the flow of lymphatic fluid and the occurrence of associated complications. ${ }^{17}$ The rate of spontaneous resolution for those with a venous component has been estimated at $3 \% .{ }^{4}$ Prenatal management options are limited and involve prenatal needle aspiration to reduce volume before delivery. ${ }^{5,18,19}$ In utero sclerotherapy treatment has also been reported. ${ }^{20}$ Location in the head and neck region may interfere with the oropharyngeal cavity. In one case series, two ex utero intrapartum treatment (EXIT) procedures were performed for fetuses with large anterior cervical malformation (intubation and tracheostomy), allowing for achievement of airway patency before cord clamping. 6

Antepartum management and mode of delivery should be individualized. Fetal hydrops is more likely to occur with lesions diagnosed early in gestation. ${ }^{5}$ There is no standardized recommendation for prenatal management. In our case, we started weekly surveillance with ultrasound Doppler studies, fluid assessment, and nonstress test at 32 weeks' gestation.

The gestational age and mode of delivery should also be individualized as there is no general recommendation. A large anterior cervical malformation, which may require an EXIT procedure, precludes vaginal delivery. Shoulder dystocia should be anticipated with vaginal deliveries. Literature review of case series suggests that prenatally diagnosed cases tend to be delivered by cesarean section, whereas missed diagnoses tend to deliver vaginally. ${ }^{5,7}$ Our patient was delivered via elective cesarean section at 39 weeks' gestation due to the size of the malformation.

Surgical resection is the mainstay of postnatal therapy of massive lymphatic malformations. Sclerotherapy with various agents (bleomycin, doxycycline, acetic acid, ethanol, Picibanil/ OK-432, EthiblocTM, sodium tetradecyl sulfate/hypertonic saline, etc.) may be used as an adjunct or alternative, especially in cases with multiple loculations and extensive disease. Use of intralesional sclerotherapy in macrocystic lymphatic malformations has shown significant efficacy and, in some cases, eliminated the need for other forms of therapy. The lack of randomized controlled trials to compare these agents and the lack of a standardized dosing protocol have hindered treatment plans. Postnatal surgical management largely depends on the size and location of the lesion. A retrospective chart review of 117 children with lymphatic malformations of the oral and cervicofacial regions, cervicofacial regions which were surgically resected, showed an overall recurrence rate or failure rate of $24 \%{ }^{21}$ Published recurrence rates range between 18 and $56 \% .^{22-24}$ In our case, a combination of postnatal resection and sclerotherapy was used. Regardless of what mode of therapy is ultimately chosen, a multidisciplinary approach, as was the case in our patient, is invaluable in the comprehensive evaluation and formulation of an appropriate treatment plan.

\section{References}

1 Mulliken JB, Young A. Vascular Birthmarks: Hemangiomas and Malformations. Philadelphia, PA: WB Saunders; 1988

2 Burrows PE, Mason KP. Percutaneous treatment of low flow vascular malformations. J Vasc Interv Radiol 2004;15(5): 431-445

3 Sherer DM, Perenyi AR, Glick SA, et al. Prenatal sonographic findings of extensive low-flow mixed lymphatic and venous malformations. J Ultrasound Med 2006;25(11):1469-1473

4 Fonkalsrud EW. Congenital malformations of the lymphatic system. Semin Pediatr Surg 1994;3(2):62-69

5 Suzuki N, Tsuchida Y, Takahashi A, et al. Prenatally diagnosed cystic lymphangioma in infants. J Pediatr Surg 1998;33(11): 1599-1604

6 Marler JJ, Fishman SJ, Upton J, et al. Prenatal diagnosis of vascular anomalies. J Pediatr Surg 2002;37(3):318-326

7 Kaminopetros P, Jauniaux E, Kane P, Weston M, Nicolaides KH, Campbell DJ. Prenatal diagnosis of an extensive fetal lymphangioma using ultrasonography, magnetic resonance imaging and cytology. Br J Radiol 1997;70(835):750-753

8 Ruano R, Aubry JP, Simon I, et al. Prenatal diagnosis of a large axillary cystic lymphangioma by three-dimensional ultrasonography and magnetic resonance imaging. J Ultrasound Med 2003; 22(4):419-423

9 Yang JI, Kim HS, Ryu HS. Prenatal sonographic diagnosis of KlippelTrenaunay-Weber syndrome: a case report. J Reprod Med 2005; 50(4):291-294

10 Chen CP, Lin SP, Chang TY, et al. Prenatal sonographic findings of Klippel-Trénaunay-Weber syndrome. J Clin Ultrasound 2007; 35(7):409-412

11 Richards DS, Williams CA, Cruz AC, Hendrickson JE. Prenatal sonographic findings in a fetus with Proteus syndrome. J Ultrasound Med 1991;10(1):47-50

12 Nahm WK, Moise S, Eichenfield LF, et al. Venous malformations in blue rubber bleb nevus syndrome: variable onset of presentation. J Am Acad Dermatol 2004;505, Suppl):S101-S106

13 Puig S, Casati B, Staudenherz A, Paya K. Vascular low-flow malformations in children: current concepts for classification, diagnosis and therapy. Eur J Radiol 2005;53(1):35-45

14 Sapp JC, Turner JT, van de Kamp JM, van Dijk FS, Lowry RB, Biesecker LG. Newly delineated syndrome of congenital lipomatous overgrowth, vascular malformations, and epidermal nevi (CLOVE syndrome) in seven patients. Am J Med Genet A 2007; 143A(24):2944-2958

15 Alomari AI. Characterization of a distinct syndrome that associates complex truncal overgrowth, vascular, and acral anomalies: a descriptive study of 18 cases of CLOVES syndrome. Clin Dysmorphol 2009;18(1):1-7

16 Clove syndrome community; Available at: http://www.clovessyndrome.org/index.cfm. Accessed on September 23, 2013 
e42 Extensive Fetal Congenital Subcutaneous Mixed Venous Lymphatic Lesion Odibo et al.

17 Mulliken JB. Vascular anomalies. In: Aston SJ, Beasley RW, Thorne CHM, eds. Grabb and Smith Plastic Surgery. 5th ed. NY: Lippincott Raven Publishers; 1997

18 Alomari AI, Karian VE, Lord DJ, Padua HM, Burrows PE. Percutaneous sclerotherapy for lymphatic malformations: a retrospective analysis of patient-evaluated improvement. J Vasc Interv Radiol 2006;17(10):1639-1648

19 Deshpande P, Twining P, O'Neill D. Prenatal diagnosis of fetal abdominal lymphangioma by ultrasonography. Ultrasound Obstet Gynecol 2001;17(5):445-448

20 Watari H, Yamada H, Fujino T, et al. A case of intrauterine medical treatment for cystic hygroma. Eur J Obstet Gynecol Reprod Biol 1996;70(2):201-203
21 Lei ZM, Huang XX, Sun ZJ, Zhang WF, Zhao YF. Surgery of lymphatic malformations in oral and cervicofacial regions in children. Oral Surg Oral Med Oral Pathol Oral Radiol Endod 2007;104(3):338-344

22 Ricciardelli EJ, Richardson MA. Cervicofacial cystic hygroma. Patterns of recurrence and management of the difficult case. Arch Otolaryngol Head Neck Surg 1991;117(5):546-553

23 Padwa BL, Hayward PG, Ferraro NF, Mulliken JB. Cervicofacial lymphatic malformation: clinical course, surgical intervention, and pathogenesis of skeletal hypertrophy. Plast Reconstr Surg 1995;95(6):951-960

24 Churchill P, Otal D, Pemberton J, Ali A, Flageole H, Walton JM. Sclerotherapy for lymphatic malformations in children: a scoping review. J Pediatr Surg 2011;46(5):912-922 